\title{
INCIDENCE OF IMPAIRED GLUCOSE METABOLISM IN PATIENTS STARTED ON TREATMENT FOR HIV AND PTB CO-INFECTION
}

\author{
P. V. Kalyan Kumar ${ }^{1}$, Ramakrishna Gorantla ${ }^{2}$, Ramakrishna Rachakonda ${ }^{3}$ \\ ${ }_{1}^{1}$ Assistant Professor, Department of Respiratory Medicine, Katuri Medical College, Guntur, AP. \\ ${ }^{2}$ Associate Professor, Department of General Medicine, Katuri Medical College, Guntur, AP \\ 3Professor, Department of Pulmonary Medicine, Katuri Medical College, Guntur, AP.
}

ABSTRACT

\section{BACKGROUND}

A high proportion of HIV patients in India are having pulmonary tuberculosis. The combined therapy of both HIV + PTB causes many unwanted side effects. The following study mainly aimed to study the impaired glucose metabolism in those patients. The aim of the study is to determine the incidence of disorders of glucose metabolism in HIV-1 infected tuberculosis adult on antiretroviral therapy (ART) and antituberculosis therapy (ATT) admitted in tertiary health centre in Guntur, Andhra Pradesh, India.

\section{MATERIALS AND METHODS}

This is two-year observational, analytical, prospective cohort study using data of all the HIV + PTB co-infected people admitted in tertiary health centre from March 2015 to March 2017. HIV infected ART naive tuberculosis patients eligible for ATT and ART (HIV-ART + ATT, $n=75$ ) are included in the study. All participants had demographic, anthropometric, biochemical and radiological assessments at baseline; in addition, the HIV-ART group had follow-up assessments for 24 months on ART. Disorders of glucose metabolism namely diabetes mellitus (DM), impaired glucose tolerance and impaired fasting glucose were defined using WHO criteria at baseline 3, 6, 12, 18 and 24 months. Poisson approximations estimated incidence of disorders of glycaemia.

Study Design- The study was a prospective, observational, analytical cohort study for two years among HIV and PTB co-infected patients.

\section{RESULTS}

At baseline, the prevalence of DM was $0 \%$ in HIV-ART + ATT patients. During the follow-up using glucose-based WHO criteria, 5 patients developed DM during 150.2 person-years with an incidence rate of 3.3 cases per 100 PYFU (95\%, CI 0.6 - 5.1). 8 participants developed IGT during 156.1 PYFU with an incidence of 5.1 cases per 100 PYFU [95\%, CI 3.5 - 6.2]) and 5 participants developed IFG during 141.5 PYFU with an incidence of 3.5 cases per 100 PYFU [95\%, CI 1.4 - 4.6]).

\section{CONCLUSION}

ART was associated with increased incidence of impaired glucose metabolism. These findings underscore the importance of clini cal monitoring of patients on ART and ATT.

\section{KEYWORDS}

Antiretroviral Therapy (ART), Antituberculosis Treatment (ATT), Pulmonary Tuberculosis (PTB), Diabetes Mellitus.

HOW TO CITE THIS ARTICLE: Kumar PVK, Gorantla R, Rachakonda R. Incidence of impaired glucose metabolism in patients started on treatment for HIV and PTB co-infection. J. Evolution Med. Dent. Sci. 2017;6(92):6613-6617, DOI: $10.14260 /$ jemds/2017/1438

\section{BACKGROUND}

HIV/ AIDS is a global pandemic. As on March 2015, there were 36.9 million (34.3 million - 41.4 million) people living with HIV, ${ }^{1}$ despite the impressive rollout of Antiretroviral Therapy (ART) programme worldwide including low- and middle-income developing countries. 1.2 million ${ }^{1}$ people died from AIDS-related causes worldwide.

A high proportion of HIV patients in India continue to be diagnosed late (CD4 count $<500$ cells $/ \mathrm{mL}$ ) with increased risk of morbidity and mortality in these population. With 2.1 million HIV infected people, India had third largest burden of

'Financial or Other Competing Interest': None.

Submission 09-08-2017, Peer Review 14-11-2017,

Acceptance 21-11-2017, Published 04-12-2017.

Corresponding Author:

Dr. P. V. Kalyan Kumar,

Assistant Professor,

Department of Respiratory Medicine,

Katuri Medical College, Guntur, AP.

E-mail:drpvkalyan@hotmail.com

DOI: $10.14260 /$ jemds/2017/1438
HIV worldwide ${ }^{2}$ and two-thirds of the patients live in rural areas. ${ }^{3}$ The total number of people living with HIV (PLHIV) in India was estimated at 21.17 lakhs (17.11 lakhs - 26.49 lakhs) in 2015 , Children ( $<15$ years) account for $6.54 \%$, while twofifth $(40.5 \%)$ of total HIV infections were females ${ }^{8}$ undivided. Andhra Pradesh and Telangana have the highest estimated number of PLHIV (3.95 lakhs) followed by Maharashtra (3.01 lakhs), Karnataka (1.99 lakhs), Gujarat (1.66 lakhs), Bihar (1.51 lakhs) and Uttar Pradesh (1.50 lakhs). These seven states together account for two-thirds (64.4\%) of total estimated PLHIV.

Guntur had the highest number of HIV positive cases since 2013 - 14. Guntur district registered 5,195 (including 2,498 female) HIV positive cases during 2013 - 2014 and 6,027 cases (2,938 female) during the previous year. Hyderabad, which tops the HIV positive chart in Telangana state reported 3,952 (2,525 female) cases during 2013 - 14.4

Andhra Pradesh assigned to category A with more than 1\% ANC prevalence (Prevalence among pregnant women attending antenatal clinics) in district in any of the 
surveillance sites in the 3-year reference period. The following regimens are given to the patients in the study.

\section{Regimen I (a)}

Tenofovir + Lamivudine + Nevirapine. First line regimen for patients with $\mathrm{Hb}<9 \mathrm{gm} / \mathrm{dL}$ and not on concomitant ATT.

\section{Regimen II (a)}

Tenofovir + Lamivudine + Efavirenz. First line regimen for patients with $\mathrm{Hb}<9 \mathrm{gm} / \mathrm{dL}$ and on concomitant ATT.

There is a paucity of data on the prevalence of diabetes in HIV-1 infected tuberculosis patients, ART naïve patients. ${ }^{5}$ The following study mainly aims to determine the incidence of impaired glucose metabolism in these patients.

\section{MATERIALS AND METHODS}

The World Health Organisation (WHO) and 2017 American Diabetes Association (ADA) criteria ${ }^{6}$ for disorders of glucose metabolism were used to determine the incidence of diabetes mellitus (DM), impaired glucose tolerance (IGT) and impaired fasting glucose (IFG) after commencing combination antiretroviral therapy (ART) and antituberculosis therapy.

\section{SAMPLING}

Sample size was taken for convenience. The study sample consisting of HIV + PTB patients admitted during 24 months period from March 2015 to March 2017.

\section{Inclusion Criteria}

1. All patients diagnosed of HIV and pulmonary tuberculosis were included in the study.

2. All patients are ART and ATT naïve patients.

\section{Exclusion Criteria}

1. All patients aged less than 18 years were excluded from the study,

2. Pregnant women, and

3. Known diabetes mellitus patients were excluded from the study.

Study procedure includes self-administered questionnaire, a detailed history and full physical examination, laboratory and radiological tests. The following demographic information was recorded: Age, gender, marital status, highest level of education, occupation history. Medical history included the following: - History of opportunistic infections, personal and family history of diabetes.

A total of 75 patients were included in the study. All these patients were followed for two years from March 2015 to March 2017. The incidence of diabetes and impaired glucose metabolism are calculated at the end of one year and two years.

\section{Study Design}

The following study is a prospective, observational and analytical cohort study for two years among the patients admitted with HIV and PTB co-infection.

Non-probability purposive sampling mainly to include the HIV + PTB patients with impaired glucose tolerance was used in the study.

\section{Statistical Methodology}

Data analysis was done by SPSS 24.0. (SPSS, IBM, USA) were used for statistical analysis. Chi-square test was used for categorical data and check the level of significance among the two groups.

\section{RESULTS}

The results of the data are summarised and analysed using appropriate, descriptive and analytical biostatistics. The baseline characteristics of the entire cohort were recorded retrospectively. The outcomes were analysed at the end of one year and two years.

Of the 75 patients who were included in the study, there were 3,2 and 2 patients with IFG, IGT and DM respectively by the end of 1 year. By year 2 there were 2, 6 and 2 patients with IFG, IGT and DM respectively. Of the 75 patients who underwent baseline testing, 72 patients have normal blood glucose values. One patient was diagnosed to have impaired fasting glucose level and 2 patients have impaired glucose tolerance test (Fig. 1).

Of the 75 patients 20 patients were lost for follow-up; 2 patients were excluded due to pregnancy and 4 patients died. One patient who was having impaired fasting glucose remained the same even after one year of followup (Fig. 2).

Of the five patients who developed DM by the end of the study period, 1 subject had IGT at baseline and DM at 3 months. Four subjects had normal OGTT at baseline. Of these, one developed DM at 6 months, two subjects had IFG during $1^{\text {st }}$ year and one patient developed impaired glucose tolerance test at the end of one year. One subject had IGT at year 1 and DM at year 2 (Fig. 1).

Five patients developed IFG during the study period. Three of them developed IFG at year 1 ; of these 3 , one patient returned to normal OGTT at year 2 and one developed DM at year 2; one remained IFG at year 2, so one patient had IFG at baseline with IFG persisting at year 2 (Fig. 1 and Fig. 2).

Of the 8 patients that developed IGT during the 2-year study period, 2 had IGT at baseline - 1 persisted as IGT by year 1, 1 developed DM at year 1 and 2 had normal OGTT at year 1 . Five patients had normal OGTT at 1 year, developed IGT at the end of $2^{\text {nd }}$ year.

Of the 2 patients having impaired glucose tolerance test at the end of 1 year, 1 patient developed diabetes mellitus at the end of one year and 1 patient remained IGT even after the end of $2^{\text {nd }}$ year (Fig. 2).

\section{Incidence of Disorders of Glucose Metabolism}

Time at risk was calculated "from ART initiation date" to the estimated date of DM, IGT and IFG. Impaired glucose regulation causing pre-diabetes (IGT or IFG) was calculated. Poisson approximations were used to calculate confidence intervals (CIs) for incidence of diabetes mellitus, IGT and IFG. Table 2 shows the incidence rate of DM, IGT and IFG in the patients taking both ATT and ART.

Of 75 HIV + TB co-infected persons that were commenced on ART + ATT, 5 developed DM during 150.2 person-years follow-up (PYFU) using glucose based WHO criteria with an incidence rate of 3.3 cases per 100 PYFU (95\%, CI 0.6 - 5.1) (Table 1). Eight participants developed IGT during 156.1 PYFU (incidence: 5.1 cases per 100 PYFU [95\%, CI 3.5 - 6.2]) and 5 participants developed IFG during 141.5 PYFU (incidence: 3.5 cases per 100 PYFU [95\%, CI 1.4 - 4.6]). 
Thirteen persons developed IGR (IGT or IFG) by glucose based WHO criteria with incidence of 8.5 cases per 152.1 PYFU [95\%, CI 3.9 - 11.2). Of 75 persons followed up, 18 developed any disorders of glucose (DM or IGT or IFG) during 161.6 PYFU (incidence: 11.1 cases per 100 PYFU [95\%, CI 5.5 - 14.3).

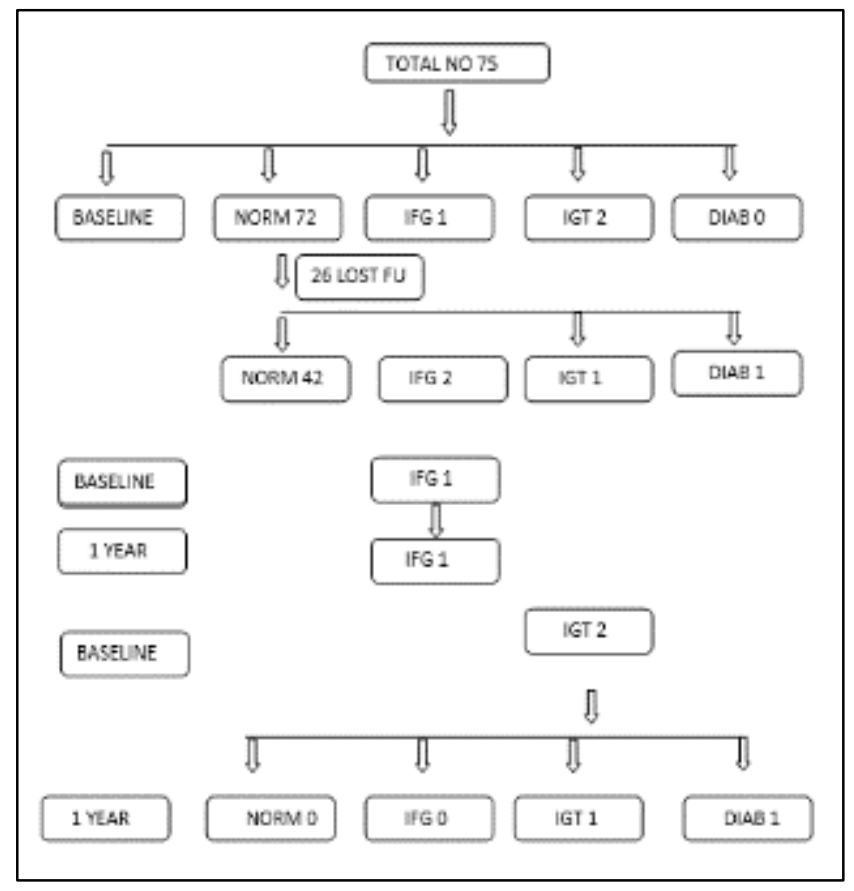

Figure 1. At One Year Follow-Up

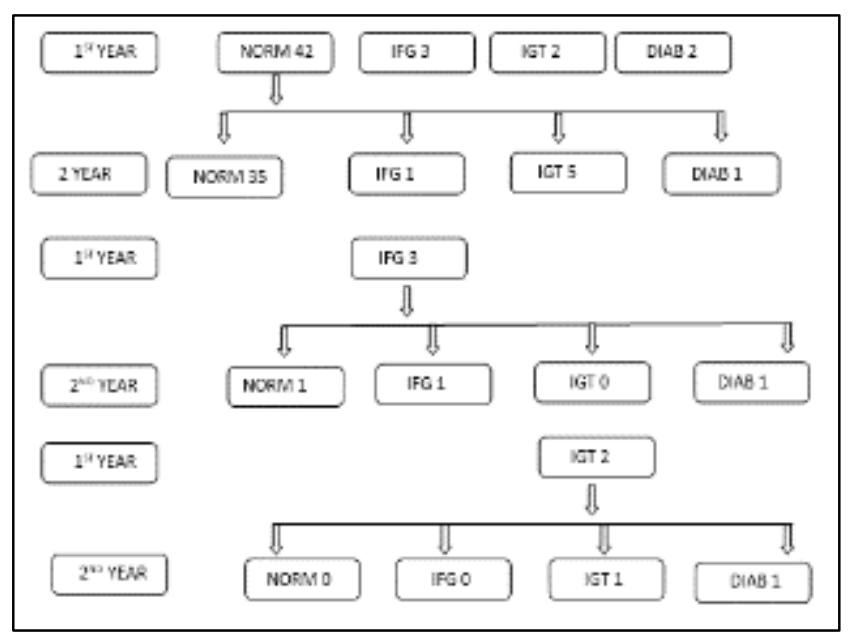

Figure 2. At 2 Years Follow-Up

\begin{tabular}{|c|c|c|c|}
\hline $\begin{array}{c}\text { Impaired } \\
\text { Glucose } \\
\text { Metabolism }\end{array}$ & $\begin{array}{c}\text { No. of } \\
\text { Patients }\end{array}$ & $\begin{array}{c}\text { No. of Person } \\
\text { Years }\end{array}$ & $\begin{array}{c}\text { Incidence/ } \\
\text { 100 Person } \\
\text { Years }\end{array}$ \\
\hline DM & 5 & 150.2 & $3.3(0.6-5.1)$ \\
\hline IFG & 5 & 141.5 & $3.5(1.4-4.6)$ \\
\hline IGT & 8 & 156.1 & $5.1(3.5-6.2)$ \\
\hline \multicolumn{2}{|c|}{ Table 1. Incidence of DM and Impaired Glucose Metabolism } \\
\hline
\end{tabular}

Table 1- Incidence of DM and impaired glucose metabolism. Chi-square test for the level of significance was used in our study. Impaired glucose metabolism is significant in HIV + TB with confidence of $95 \%$ with $\mathrm{x}^{2}$ value of 32.68 ( $\mathrm{p}$ value < 0.05).

\section{DISCUSSION}

This study has investigated impaired glucose metabolism in an ethnically homogeneous, HIV-1 infected tuberculosis population. At baseline using both World Health Organisation (WHO) and American Diabetes Association (ADA) glucosebased criteria, none of the HIV-1 tuberculosis infected subjects (whether or not initiated on ART) had diabetes. There is a paucity of data on the prevalence of diabetes in HIV-1 infected tuberculosis patients, ART naïve patients. ${ }^{7}$

Low diabetes prevalence in HIV infected, ART naïve patients has previously been reported. ${ }^{8}$ Kilby et al ${ }^{7}$ found a prevalence of hyperglycaemia of $1.9 \%$ in ART naïve HIV infected patients, after reviewing blood glucose results from medical records. This was probably the first report of the frequency of hyperglycaemia in HIV infected patients, but this study did not include pulmonary tuberculosis patients. In a subset of those who developed diabetes in that study had been treated with megestrol for weight loss, so there was an association between hyperglycaemia and megestrol use. Although, not directly comparable in that study. In other study, El-Sadr et $\mathrm{al}^{8}$ reported that more advanced severe HIV disease was associated with less favourable glucose homeostatic profile.

Consistent with the findings in this study, Galli et $\mathrm{al}^{9}$ reported diabetes prevalence of $0.8 \%$ in a subset of HIV infected ART-naïve subjects. By contrast, the Multicentre AIDS Cohort Study (MACS) found a higher prevalence among HIV-infected, ART naïve men ${ }^{10}$; but in that study the rate of diabetes in HIV negative controls was similar to the current study.

The prevalence of diabetes in HIV infected ART naïve subjects in the present study is consistent with that found in previous studies, although demographic characteristics differ between these studies. The majority of available data in cohorts are predominantly men, Caucasian and in developed countries. The present study was set in a developing country like India. The significance of the ethnic distribution is that it has been postulated that Indian people may metabolise antiretroviral drugs and antituberculosis drugs differently and because the background prevalence may be different; also, ART and ATT together may have been shown to influence insulin sensitivity.

Demographic characteristics (older age, Asian ethnicity, male gender), higher body mass index, lower serum HDL cholesterol and higher serum triglyceride are factors that have been associated with diabetes in HIV infected patients as in the general population. However, there is paucity of data in developing countries.

The low prevalence of diabetes in ART and ATT naïve subjects in the present study and other studies suggests a relatively low association between HIV infection and diabetes perhaps on the basis of reduction in BMI associated with HIV infection.

\section{Incidence of Glycaemic Disorders}

This study describes for the first time in India to find out the incidence of diabetes mellitus (DM), impaired glucose tolerance (IGT), impaired fasting glucose (IFG) among subjects initiated on ART + ATT and followed up prospectively over 24 months. As stated above, at baseline there was an absence $(0 \%)$ of diabetes in HIV-ART. 
Of 75 HIV + TB co-infected persons that were commenced on ART + ATT, 5 developed DM during 150.2 person-years followup (PYFU) using glucose based WHO criteria with an incidence rate of 3.3 cases per 100 PYFU (95\%, CI 0.6 - 5.1) (Table 1). Eight participants developed IGT during 156.1 PYFU (incidence: 5.1 cases per 100 PYFU [95\%, CI 3.5 - 6.2]) and 5 participants developed IFG during 141.5 PYFU (incidence: 3.5 cases per 100 PYFU [95\%, CI 1.4 - 4.6]). Thirteen persons developed IGR (IGT or IFG) by glucose based WHO criteria with incidence of 8.5 cases per 152.1 PYFU [95\%, CI 3.9 - 11.2). Of 75 persons that followed up, 18 developed any disorders of glucose (DM or IGT or IFG) during 161.6 PYFU (incidence: 11.1 cases per 100 PYFU [95\%, CI 5.5 - 14.3).

\section{CONCLUSION}

The incidence of DM and impaired glucose tolerance are higher among HIV and PTB co-infected patients started on the treatment.

The following study helps to check the diabetic status of the co-infected patients regularly three times annually, if they were started on dual ART and ATT treatment.

\section{REFERENCES}

[1] UNAIDS, Report on the global AIDS epidemic, 2010. http://www.unaids.org/globalreport/Global_report.ht ml. Accessed on 2017 Aug 08.

[2] UNAIDS/WHO, Country Progress Report, UNGASS, India, March 2014. http://www.unaids.org/en/dataanalysis/knowyourre sponse/countryprogressreports/2014countries.Acces sed on 2017 Aug 08.
[3] UNAIDS/WHO, Country Progress Report, UNGASS, India, $\quad$ March 2010. data.unaids.org/pub/report/2010/india_2010_countr y_progress_report_en.pdf. Accessed on 2017 Aug 08.

[4] Alvarez-Uria G. Factors associated with delayed entry into HIV medical care after HIV diagnosis in a resource-limited setting: data from a cohort study in India. Peer J 2013;1:e90.

[5] Brar I, Shuter J, Thomas A, et al. A comparison of factors associated with prevalent diabetes mellitus among HIV-infected antiretroviral-naive individuals in the National Health And Nutritional Examination Survey Cohort. J Acquir Immune Defic Syndr 2007;45(1):66-71.

[6] Kumar R, Nandhini LP, Kamalanathan S, et al. Evidence for current diagnostic criteria of diabetes mellitus. World J Diabetes 201615;7(17):396-405.

[7] Kilby JM, Tabereauc PB. Severe hyperglycaemia in an HIV clinic: preexisting versus drug-associated diabetes mellitus. J Acquir Immune Defic Syndr and Human Retrovirology 1998;17(1):46-50.

[8] El-Sadr WM, Mullin CM, Carr A, et al. Effects of HIV disease on lipid, glucose and insulin levels: results from a large antiretroviral-naive cohort. HIV Medicine 2005;6(2):114-21.

[9] Galli L, Salpietro S, Pellicciotta G, et al. Risk of type 2 diabetes among HIV-infected and healthy subjects in Italy. Eur J Epidemiol 2012;27(8):657-65.

[10] Brown TT, Cole SR, Li X, et al. Antiretroviral therapy and the prevalence and incidence of diabetes mellitus in the multicenter AIDS cohort study. Arch Intern Med 2005;165(10):1179-84. 